\title{
CELLULAR ROBOTICS AND \\ MICRO ROBOTIC SYSTEMS
}


World Scientific Series in Robotics and Automated Systems - Vol. 10

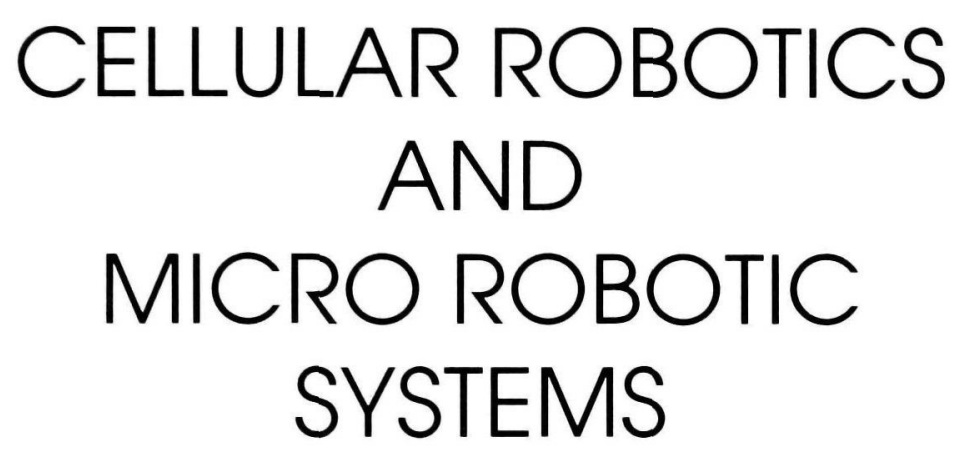

T FUKUDA \& T UEYAMA

Department of Mechano Informatics and Systems Nagoya University Japan

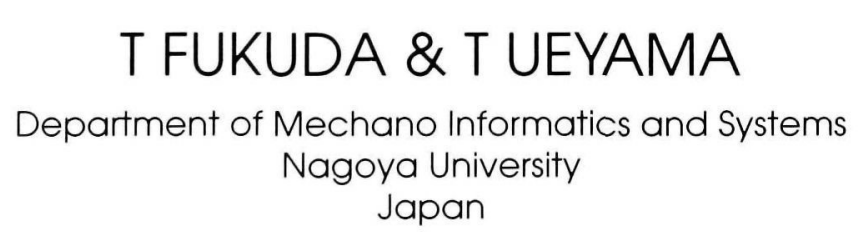




\section{Published by}

World Scientific Publishing Co. Pte. Ltd.

P O Box 128, Farrer Road, Singapore 9128

USA office: Suite 1B, 1060 Main Street, River Edge, NJ 07661

UK office: 73 Lynton Mead, Totteridge, London N20 8DH

\section{CELLULAR ROBOTICS AND MICRO ROBOTIC SYSTEMS}

Copyright () 1994 by World Scientific Publishing Co. Pte. Ltd.

All rights reserved. This book, or parts thereof, may not be reproduced in any form or by any means, electronic or mechanical, including photocopying, recording or any information storage and retrieval system now known or to be invented, without written permission from the Publisher.

For photocopying of material in this volume, please pay a copying fee through the Copyright Clearance Center, Inc., 27 Congress Street, Salem, MA 01970, USA.

ISBN: $981-02-1457-\mathrm{X}$ 


\section{Preface}

This book presents an advanced robotic technology including two topics, cellular robotics and micro robotic systems. Both two topics have some common research issues, such as miniaturization, decentralization, and development of autonomous robots. These common researches are derived from the characteristics of cellular robotics and micro robotic systems. Since a fundamental component or robotic unit is designed as a simple function in cellular robotics, miniaturization of the components or robotic units is suitable to cellular robotics. On the other hand, the miniaturization of robots makes it difficult to provide multiple functions, therefore the provided function of a micro robot is limited, and decentralization of the micro robotic systems will be required to be functional enrichment. This book intends to present recent research results in cellular robotics and micro robotic systems. The cellular robotics aims at developing autonomous decentralized robotic systems, and building on the robotic system consisting of a large number of individual robotic units. Therefore, the miniaturization of the individual robotic units increases the application fields and the performance activities. The cellular robotic system (CEBOT) was proposed in 1987 as a dynamically reconfigurable robotic system. The CEBOT is one of the robotic systems based on the cellular robotics. Former idea has been proposed in 1983 named CCV (Control Configured Vehicle). The CCV has flexible mechanism to adjust the mechanical structure depending on its environment. The idea derived from the complexity of maintenance, surveillance, and flexible automation in faculty. The name of the cellular robotics comes from the similarity of biotic organization, in which a large number of natural cells work functionally. On the other hand, recently the micro robotic system has been developed, which has been based on the principle technique of fabrication of IC-based structural components, actuators, sensors, electronics on silicon. More advanced micro technology will bring more integrated and sophisticated robotic systems. The micro robot can be promising for all aspects of robotic systems from the viewpoint of miniaturization, precision, simple logic intelligence, and closely located sensory system, communication/control architecture, and Artificial Life. This technology will inevitably induce the distribution/decentralization in robotic system that has functional decentralization and distribution. The decentralization is caused by the performance ability and the productivity of the micro robotic system. The performance ability is limited according to the size, and the productivity is depending on fabrication techniques. It is necessary to develop a micro robotic system as an autonomous robotic system, that is, the demand is caused from the controllability of the micro robots. The controllability refers to sensitivity of the robots. The high sensitivity will be demanded in the micro robotic system, since the system sensitivity, which is caused by scale effect, is proportional to decreasing the dimension of the system generally. The accompaniment of autonomous activity to the micro robotic system is an effective method to control the system responsibly, and to decentralize the system.

Part I introduces the reason that decentralization and miniaturization in robotics are demanded. The decentralizing phenomenon occurs in whole world, such as in politics and economics. Especially, the decentralization of computer system has been developed in computer science. In the same way, in robotics the decentralization of robotic systems has been in progress because of the increasing of the complexity of the system and the developing of micro computer devices. From the view point of robotic application, the first chapter shows the purpose of developing the decentralized and micro robotic systems. Then, the relationship between two themes, presented in this book, cellular robotics and micro robotic systems is denoted from the standpoint of these characteristics. Advantage and developing issues to realize the decentralized robotic systems are also described. As 
the first discussion, single robot and multiple robots are compared in section 2.1, where the multiple robots refer to the decentralized robotic system. Since the multiple robotic system includes more than two robots in the system, coordination and cooperation have to be considered to perform any task. In section 2.2 , the second discussion presents communication issues for the multiple robots to carry out any task with coordination and cooperation in the system. The effectiveness or the necessity of coordination and cooperation in the decentralized robotic system are described in section 2.3. The coordination and cooperation are observed in human society. That is, the decentralized robotic system has characteristics of the same issue in human society. The idea extends to robotic society, which is introduced in section 2.4 .

In Part II, the concept of cellular robotics and related research works are introduced. The concept relates to the idea of robotic society, since robotic systems based on the cellular robotics consist of a large number of autonomous robots named cells. The name of the cell comes from an analogy with a cell in creature. Authors have researched a cellular robotic system (CEBOT). The CEBOT is one of the robotic systems based on the cellular robotics. In this part, several research works are introduced, such as communication problems, network architecture problems, decentralized decision making problems, and structural formation problems. At first, chapter 4 presents the communication problem. The communication problem is one of the most important issues for decentralized robotic systems, since the performance of the robotic systems depends on the effectiveness of communication in the system. This chapter deals with the analysis and the evaluation of communication amount among the cell. According to the analysis, a knowledge allocation method is introduced. The knowledge refers to the information to control robotic units or cells. As a result of proposed method, the decrease of the communication amount is shown. As the second issue, chapter 5 describes an issue of network architecture, which relates to the communication problem. The network is organized by many cells. The network architecture problem, which is treated in this chapter, is based on the communication or control effectiveness in the system. To evaluate the network architecture, an evaluation function is proposed. The evaluation function forms an energy function. And the simulation results show the grouping according to the decrease of the energy value. Chapter 6 describes a cooperative decision making that is demanded to control the decentralized systems consisting of multiple autonomous robots or multiple agents. First, a genetic algorithm is briefly introduced. Second, a decentralized intelligent system named cellular intelligence is presented, which has evolutional ability based on the genetic algorithm. Then, a genetic algorithm is applied as the cooperative decision making method, where the genetic algorithm is modified to the decentralized robotic systems such as the CEBOT. Simulation described in the chapter represents a path planning issue to configure the structure of a cellular robot, which is one of the structural configuration problems of the CEBOT. Chapter 7 presents two issues concerning structural formation problems. The structural formation problems stem from the characteristic of dynamic reconfiguration of the structure, since the cellular robotic system consists of many cells and the structure depends on the combination of the cells. The combination depends on any task and its environment. The first one is an optimal structure determination problem of the CEBOT. As the other issue, a prototype CEBOT Mark II is introduced. The prototype was designed to practice the concept of the CEBOT, which is used to carry out a docking experiment described

In part III, several examples of micro robotic systems are introduced. Technology of the micro robotic systems is adequate for the cellular robotics, because of the relation between the micro robotic systems and the cellular robotics as above described. Main topics are control methods of the micro robotic systems, in which three types of micro robots are described. In chapter 9 , at first, the configuration of micro robots is discussed 
from the view point of scale advantage or disadvantage. The actuators for the micro robots are different from conventional robots, since it is necessary to consider the influence of minute dimension. Second, the fundamental technology of the micro robotic system is described. The micro robotic system is one of the most advanced robotic systems, and has been developed in $1980 \mathrm{~s}$, which is based on silicon process. The micro robotic systems will be applied and developed in many fields. Finally, the topic of application fields is discussed. The application of micro robots as multiple robots is based on scale advantage or disadvantage, and productivity. That is, the micro robots supplement the limited power and function with group organization of them to carry out given tasks. The multiple micro robots will increase the application fields and extend the fields to unexpected fields. In chapter 10, typical microfabrication methods are introduced briefly. The technique to product the micro robots is almost based on the technique of silicon process. In the following chapters, the control methods of micro robotic systems are presented, which are dexterous control, high precision control, and non-contact control. The dexterous and high precision control methods are adapted to micro manipulators. In these control methods, the influence of nonlinearity is considered. The issue of nonlinearity is an important issue for the control of micro robots, such as nonlinear term of actuators or friction. The third control method introduced in chapter 13 has a great advantage to control micro robots. As a noncontact control method, optical control is introduced. The micro robot is controlled by ultraviolet ray, which includes both the transmission of information and the supply of energy. The non-contact control will extend the capability of micro robots extremely. 


\section{Contents}

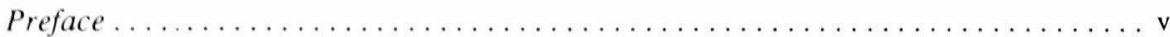

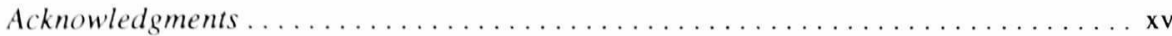

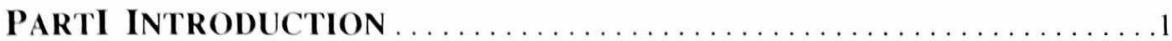

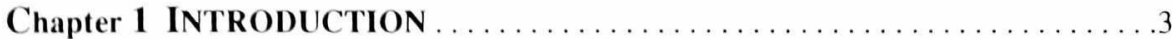

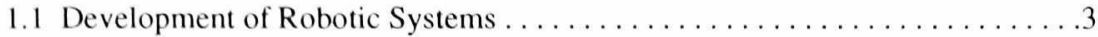

1.2 Decentralization and Miniaturization $\ldots \ldots \ldots \ldots \ldots \ldots \ldots \ldots \ldots \ldots \ldots \ldots \ldots \ldots \ldots$

1.3 Summary . . . . . . . . . . . . . . . . . . . . . . . . . . . 9

Chapter 2 DECENTRALIZED ROBOTIC SYSTEM $\ldots \ldots \ldots \ldots \ldots \ldots \ldots \ldots$

2.1 From Single Robot to Multiple Robots ......................

2.1.1 Required Characteristics on Robots .......................11

2.1.2 Advantages of Multiple Robots .......................12

2.1.3 Additional Requirement for Multiple Robots . . . . . . . . . . . . 15

2.2 Communication ......................................

2.2.1 Communication and Multiple Robots .................... 15

2.2.2 Required Characteristics of Communication .................. 16

2.3 Coordination and Cooperation ..............................

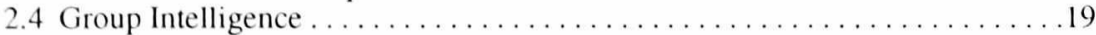

2.4.1 What is Group Intelligence? ......................... 19

2.4.2 Characteristics of Group Intelligence ..................... 20

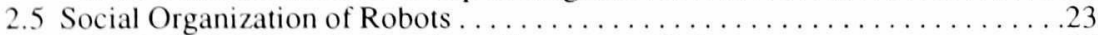

2.5.1 Social Organization from Creatures to Robots ..................23

2.5.2 Artificial Life and Decentralized Robotic System ...............25

2.5.3 Analysis and Imitation of Biological Mechanism ..............25

2.5.4 Artificial Creatures Based on Biological Models ................26

2.5.5 Classification of Research on Artificial Life ....................28

2.6 Summary . . . . . . . . . . . . . . . . . . . . . . . . . . . . . . 29

PARTII Cellular Robotics. . . . . . . . . . . . . . . . . . . . 31

Chapter 3 CONCEPT OF CEllular ROBOTICS . . . . . . . . . . . . . . . 33

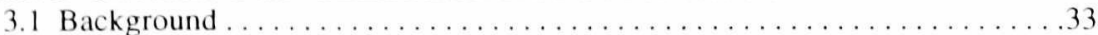

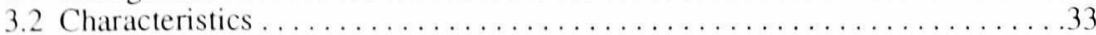

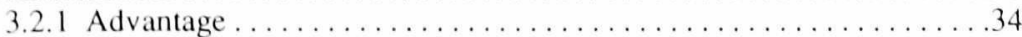

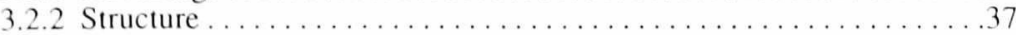

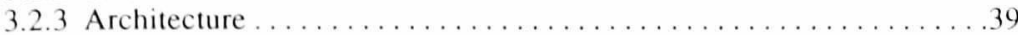

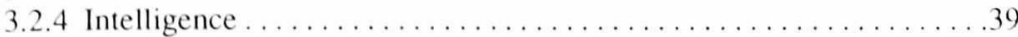

3.2 .5 Control . . . . . . . . . . . . . . . . . . . . . . . . . . . . . .39

3.3 Related Research . . . . . . . . . . . . . . . . . . . . . . . . . . . . 40

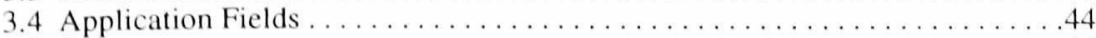

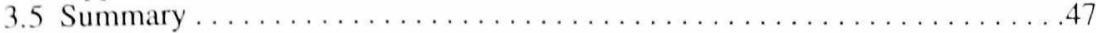


Chapter 4 CommuniCATION Issues . . . . . . . . . . . . . . . . . . 49

4.1 Necessity of Communication among cells ...................... 49

4.1.1 Necessity of Communication in Undocked State . . . . . . . . . . . . . 49

4.1.2 Necessity of Communication in Docked State. . . . . . . . . . . . . . 49

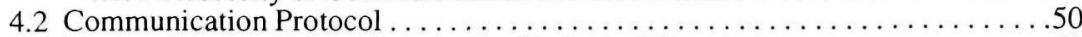

4.2.1 Communication Protocol in Undocked State .................50

4.2.2 Communication Protocol in Docked State . . . . . . . . . . . . . . 52

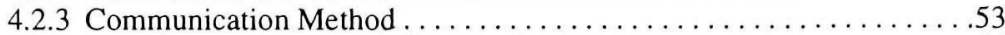

4.2.4 Experimental Results of Communication . . . . . . . . . . . . . . . 56

4.3 Analysis of Communication Amount ..............................59

4.3.1 Communication Between Cells .......................6 60

4.3.2 Estimation of Cell Communication Frequency .................63

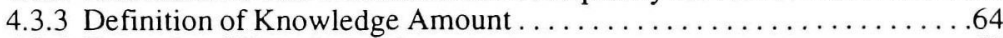

4.3.4. Example of Knowledge Amount .........................67

4.3.5 Optimal Knowledge Allocation Method .................... 70

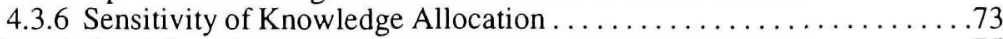

4.4 Knowledge Allocation ................................... 75

4.4.1 Matrix Notation for Distributed System ...................76

4.4.2 Knowledge Allocation Method and Algorithm ............... 80

4.4.3 Simulation Results . . . . . . . . . . . . . . . . . . . . . . . . . 83

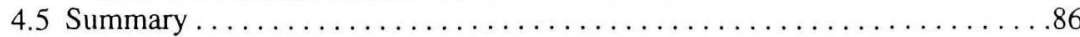

Chapter 5 NETWORK ORganization . . . . . . . . . . . . . . . . . . . . . . 87

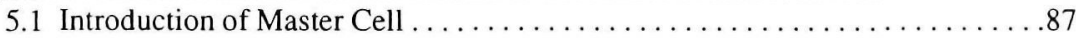

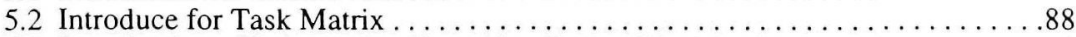

5.3 Introduction of Information Transition Probability Matrix $\ldots \ldots \ldots \ldots \ldots \ldots .90$

5.3.1 Active Probability Distribution for Cells .................. 90

5.3.2 Information Transition Probability Matrix ..................91

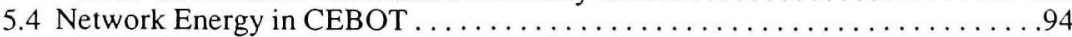

5.4.1 Stationary Distribution Vector for Information Flow . . . . . . . . . . 95

5.4 .2 Network Energy in CEBOT . . . . . . . . . . . . . . . . . . . . . 95

5.5 Mastering Using Local Energy Sensitive Estimation . . . . . . . . . . . . . . . . 96

5.6 Group Organization with Multiple Master Cells ...................98

5.7 Revised Definition of Network Energy . . . . . . . . . . . . . . . . . .99

5.7.1 Revised Definition of Information Transition Probability Matrix ..... 99

5.7.2 Revised Definition of Network Energy ...................... 100

5.8 Cross-Relation Between Cells and Task Modules ..................... 101

5.9 Evaluation of Communication Frequency between Master Cells . . . . . . . . 102

5.9.1 Communication Between Master Cells ........................ 102

5.9.2 Communication Frequency Between Master Cells ................ 102

5.9.3 Evaluation of Communication Frequency Between Master Cells . . . . 104

5.10 Search Algorithm for Multiple Master Cells ....................... 105

5.10.1 Sensitivity for Searching Master Cells ................... 105

5.10 .2 Search Algorithm ..................................... 106

5.11 Simulation Results ................................... 108

5.11.1 Low Cross-Dependence between Cells ....................108

5.11.2 Local Cross-Dependence between Cells .....................110

5.11.3 High Cross-Dependence Between Cells ......................111

5.11.4 Influence Between the Optimal Architecture and the Weight for the Cross-Dependence . . . . . . . . . . . . . . . . . . . 112 


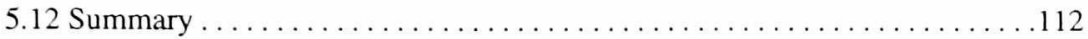

\section{Chapter 6 COOPERATIVE DECESION MAKING USING} GENETIC ALGORITHM .

6.1 Genetic Algorithms ................................... 113

6.1.1 What are Genetic Algorithms? ......................... 113

6.1.2 Related Research of Genetic Algorithms in Robotics ............ 115

6.1.3 Evolutional Cellular Intelligence Using Genetic Programing . . . . . . 117

6.2 Issue on Structural Configuration ............................... 119

6.2.1 How to Treat the Structure Presentation ...................... 119

6.2.2 How to Determine the Path Planning Cooperatively ............121

6.3 Application of Genetic Algorithm . . . . . . . . . . . . . . . . . . . . 121

6.3.1 Fitness Function ................................. 121

6.3 .2 Reproduction Rule ............................... 122

6.3.3 Crossover Operation . . . . . . . . . . . . . . . . . . . . . . . . 123

6.3 .4 Mutation Operation ................................

6.4 Distributed Genetic Algorithm ................................. 124

6.4.1 Concept of Distributed Genetic Algorithm ....................124

6.4 .2 Consideration of Communication Frequency .................. 125

6.5 Simulation Results ...................................... . . . . . . . . .

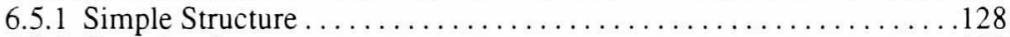

6.5 .2 Complex Structure . . . . . . . . . . . . . . . . . . . . . . 128

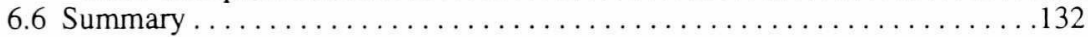

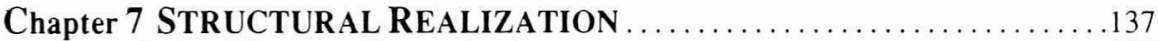

7.1 Optimal Structure Determination ........................... 137

7.1.1 Base Determination of Mobile Base Type Manipulator ........... 138

7.1.2 Determination Method for Fixed Base System .................. 140

7.1 .3 Structure Evaluation Function $\ldots \ldots \ldots \ldots \ldots \ldots \ldots \ldots \ldots \ldots \ldots \ldots \ldots$

7.1.4 Simulation Results ..................................... 149

7.2 Prototype CEBOT Mark II . . . . . . . . . . . . . . . . . . . . . . . . . 151

7.2 .1 Cell Specification . . . . . . . . . . . . . . . . . . . . . . 151

7.2 .2 Sensor System .................................... 152

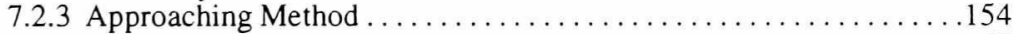

7.2 .4 Experimental Results . . . . . . . . . . . . . . . . . . . . . . . 155

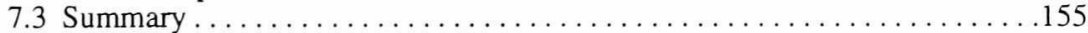

Chapter 8 CONCLUSIONS . . . . . . . . . . . . . . . . . . . . . . . . . . 157

8.1 Conclusing Remarks ...................................... 157

8.2 Future Work .........................................

PART III MICRO ROBOTIC SYSTEMS . . . . . . . . . . . . . . . . . . . 161

Chapter 9 CONCEPT OF MICRO ROBOTIC SYSTEMS . . . . . . . . . . . 163

9.1 Micro Robotic Systems and Fundamental Technology .................. 163

9.2 Research Issues on Micro Robotic Systems . . . . . . . . . . . . . . . . . . . 164

9.3 Configuration of Micro Robotic Systems . . . . . . . . . . . . . . . . . . . . . . . . 169

9.4 Application Fields ....................................... 174

9.5 Summary ............................................ 


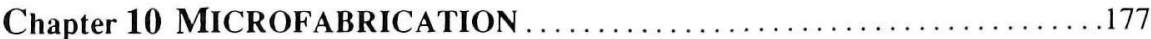

10.1 Microfabrication Based on Conventional Machining Method ............ 177

10.1. 1 Eletro Discharge Machining .........................177

10. 1.2 Wire Electro Discharge Grinding (WEDG) ................ 177

10. 1. 3 Laser Beam Machining ............................... 178

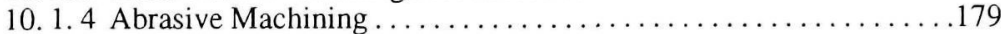

10.2 Microfabrication Based on Non-Conventional Machining Method .........179

10. 2. 1 Silicon Process ....................................... . . . . . . .

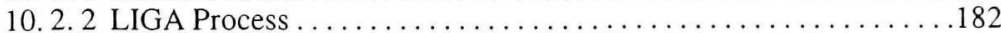

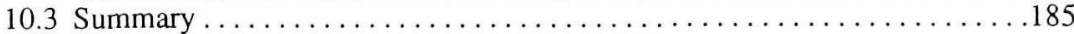

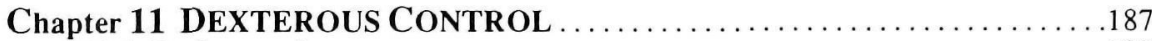

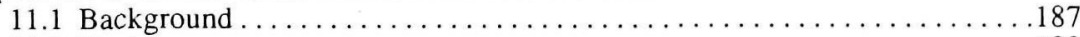

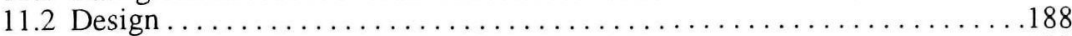

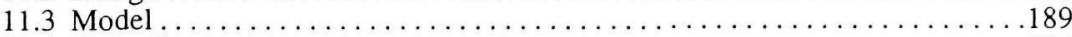

11.4 Control . . . . . . . . . . . . . . . . . . . . . . . . . . . . . . . . . . . . . 194

11.5 Experimental Results . . . . . . . . . . . . . . . . . . . . . . . . . . 197

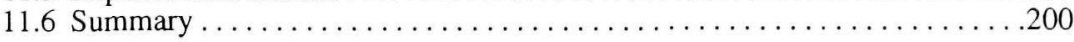

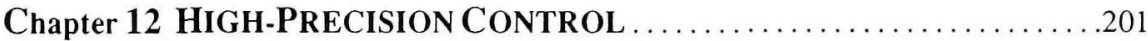

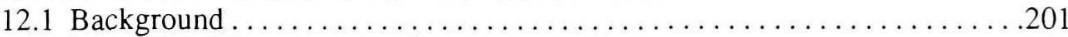

12.2 Principle of Electrostatic Micro Actuator . . . . . . . . . . . . . . . . . . . .201

12.2.1 Basic Principle I. . . . . . . . . . . . . . . . . . . . . . . . .201

12.2.2 Basic Principle II . . . . . . . . . . . . . . . . . . . . . . . . . .202

12.3 Modeling and Dynamics of 3 D.O.F. Electrostatic Micro Actuator ......... 204

12.3.1 Moving Modes ......................................204

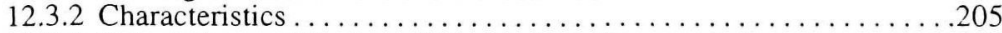

12.3.3 Calculation and Design of Mechanical Characteristics .......... 206

123.4 Dynamics ...................................210

12.4 Control Method of 3 D.O.F. Electrostatic Actuator . . . . . . . . . . . . . . . . 214

12.4.1 Position Control Method ..............................214

12.4.2 Nonlinear Feedback Control ...........................215

12.5 Position Control Simulation of 3 D.O.F. Electrostatic Micro Actuator . . . . .216

12.6 Structure of Prototype 3 D.O.F. Electrostatic Micro Actuator .............217

12.7 Experimental Results of Controlling 3 D.O.F. Electrostatic Micro Actuator

12.8 Design and Structure of 6 D.O.F. Electrostatic Actuator ................. 219

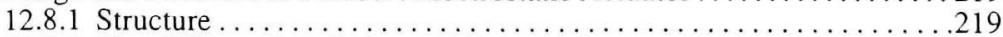

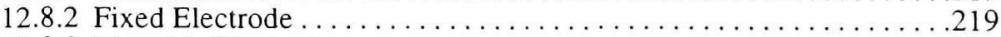

12.8.3 Movable Electrode . . . . . . . . . . . . . . . . . . . . . .220

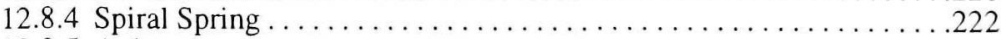

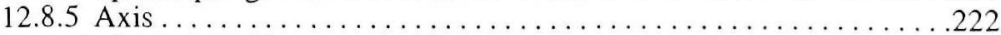

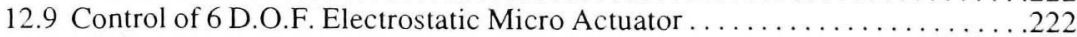

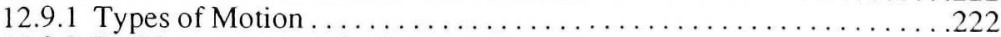

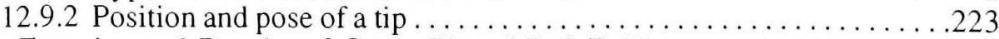

12.10 Experimental Results of Controlling 6 D.O.F. Electrostatic Micro Actuator

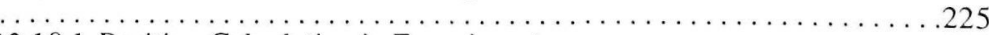

12.10.1 Position Calculation in Experiment ....................225

12.10.2 Experimental Results ..........................225 


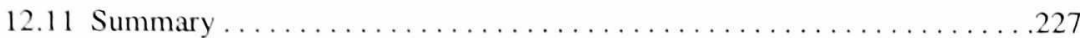

Chapter 13 NON-CONTACT CONTROL ............................229

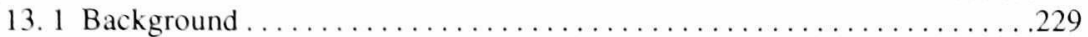

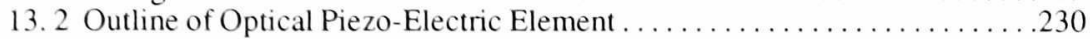

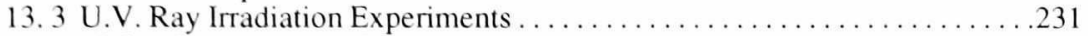

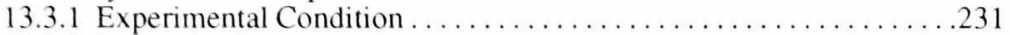

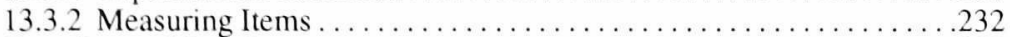

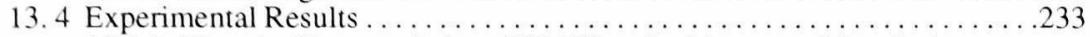

13.4.1 Electric Characteristics of PLZT at the Moment of Irradiation

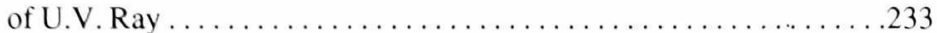

13.4.2 Time history of Generated Electromotive Current at the Moment of Irradiation of U.V. Ray .................233

13.4.3 Time History of Generated Strain by Irradiation of U.V. Ray . . . . . .235

13. 5 Photo Response Model of Optical Piezo-Electric Element

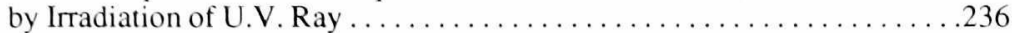

13.5.1 Basic Idea of Photo Response Model . . . . . . . . . . . . . . . . . 236

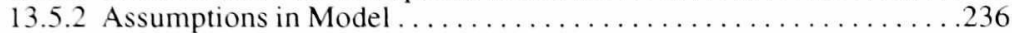

13.5.3 Derivation of Photo Response Model . . . . . . . . . . . . . . . . .237

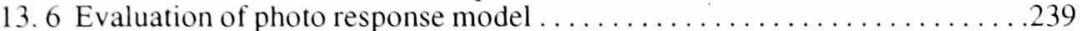

13.6.1 Identification of Function $\mathrm{P}(\lambda), \mathrm{Q}(\lambda), \operatorname{Ls}(\lambda, t) \ldots \ldots \ldots \ldots \ldots \ldots . \ldots \ldots$

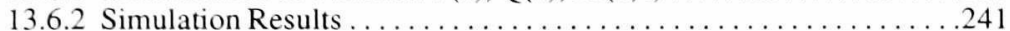

13. 7 Outline of Bimorph Optical Piezo-electric Actuator . . . . . . . . . . . . . . 242

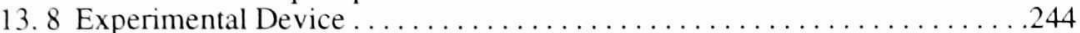

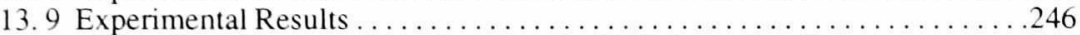

13.9.1 Time History of Displacement by U.V. Beam Irradiation . . . . . . . . 246

13.9.2 Time History of Electromotive Current by U.V. Beam Irradiation

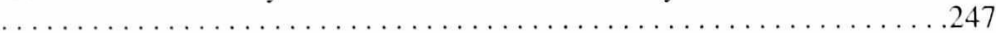

13.9.3 Time History of Displacement and Temperature by Heating . . . . . 247

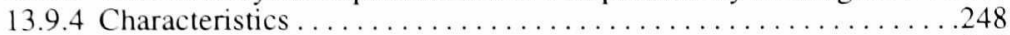

13.9.5 Optical Servo System . . . . . . . . . . . . . . . . . . . . . . . 249

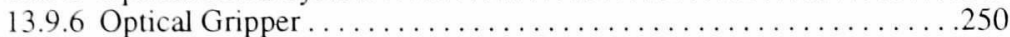

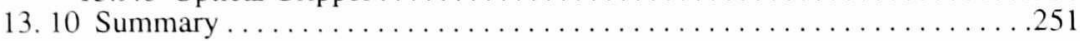

Chapter 14 Conclusions . . . . . . . . . . . . . . . . . . . . . . . . . . . 253

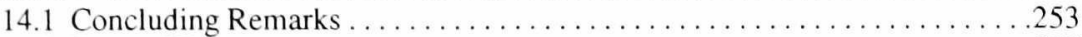

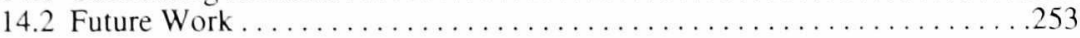

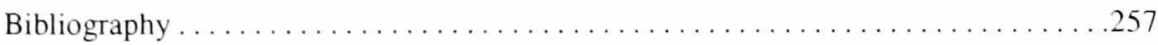

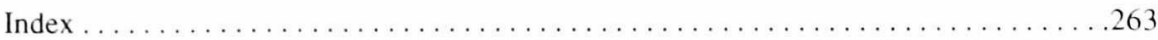




\section{Acknowledgments}

We express our gratitude to the World Scientific Publisher to take the opportunity to publish this work in this growing area. The research topics presented in this book have been investigated in our laboratory, Department of Mechano-Informatics \& systems, Nagoya University for many years. The described topics are concerned in the Cellular Robotics and Micro Robotic Systems. The concept of the Cellular Robotics that was proposed by Prof. T.Fukuda in 1987 came from the application of robots in hazardous environments, such as power plant, nuclear plant, chemical plant, and so on, because of the difficulty to work in restricted working environments, such as small entrance, large working space, heavy objects, and so on. In order to work in these conditions, it is necessary to cooperate and coordinate, and to construct optimal or feasible robots that have suitable dimensions to perform particular tasks. To adapt the robots to the environment, the robots should be constructed by a number of parts or modules, so that the robots have the ability of reconfiguration of itself according to any tasks and its environments. First, according to this idea, the reconfigurable robot had been named as a Control Configured Robot (CCR) after Control Configured Vehicle (CCV). Afterward, the research on this robotic system has renamed as the cellular robotics after the configuration of biomechanism, which consists of a huge number of cells as an example of self-organizing or self-constructing systems. In the same time, the research on Micro Robotic Systems, such as micro manipulators, has started up. The final target of the Cellular Robotics and Micro Robotic Systems is to integrate both research advantages, decentralization and miniaturization. The word of the micro robotic systems refers to micro machines and micro manipulators, and this technology includes human-machine interface. The micro machines work according to the scale advantage and carry out any tasks cooperatively because of the limitation of the functions and the productivity of the micro machines. The micro manipulators will be used to operate small objects and to manipulate high precise machining. Additionally, to operate the micro machines and the micro manipulators, it is necessary to integrate and develop the human-machine interface into the micro robotic systems. Therefore, the micro robotic systems include the micro machines and the micro manipulators, and human interface cannot be ignored to construct the micro robotic systems. Especially, micro manipulation technology is one of the important issues to translate sensing signals adequately from the world of the micro robots into the world of human operators.

Finally, we would like to thank many people, especially Dr. Y.Kawauchi, Mr. M.Fujiyoshi, Mr. S.Hattori, Mr. S.Nakagawa, and Mr. T. Tanaka, who have provided their efforts to meaningful research fulfillment. 